VOL. 52 (1995) [31-39]

\title{
POSITIVE DEFINITE FUNCTIONS AND RELATIVE PROPERTY (T) FOR SUBGROUPS OF. DISCRETE GROUPS
}

\author{
Teresa Bates and Guyan Robertson
}

\begin{abstract}
Relative Property (T) for a subgroup $B$ of a group $G$ and its connection with positive definite functions are studied. A relation with the Haagerup approximation property is established. We show that if $H$ is a non-normal subgroup of a group $G$ which has Property (T) and G/H is amenable as a graph then $H$ has finite index in $\mathbf{G}$.
\end{abstract}

\section{INTRODUCTION}

A locally compact group $G$ with a countable base has Kazhdan's Property (T) if every unitary representation of $G$ which almost has invariant vectors actually has a (non-zero) invariant vector. The following equivalent formulation [4, Chapitre 1] can be used to make this definition more precise:

$G$ is compactly generated and for any compact generating set $K$ there exists $\varepsilon>0$, such that if $\pi: G \rightarrow \mathcal{U}(\mathcal{H})$ is a unitary representation of $G$ on a Hilbert space $\mathcal{H}$ and $\xi \in \mathcal{H},\|\xi\|=1$ satisfies

$$
\sup _{g \in K}\|\pi(g) \xi-\xi\|<\varepsilon
$$

then $\pi$ has a non-zero invariant vector.

Margulis has generalised this concept as follows:

Let $H$ be a closed subgroup of $G$. The pair $(G, H)$ has Relative Property $(T)$ if every unitary representation of $G$ which almost has $G$-invariant vectors actually has a non-zero $H$-invariant vector. When $G=H$ this definition coincides with that of the usual property (T). This seemingly technical condition on a group turns out to have important applications in suprisingly diverse areas of mathematics $[4,8]$.

We begin this paper by outlining some equivalent definitions of relative property (T) and discussing its behaviour under passage to closed subgroups of finite covolume. The results obtained are directly analogous to those for the usual property ( $T$ ) found in [4].

\section{Received 30th August, 1994.}

This research was supported by the Australian Research Council.

Copyright Clearance Centre, Inc. Serial-fee code: 0004-9729/95 \$A2.00+0.00. 
In the case of discrete groups we show how positive definite functions can be used to give direct proofs of these, and other, results. In particular, we show that if a discrete group $G$ has the Haagerup approximation property then $G$ has no infinite subgroup $H$ such that $(G, H)$ has relative property (T).

Finally we show that if $G$ is a discrete group with property $(\mathrm{T})$ and $H$ is a subgroup of $G$ such that the Schreier coset graph of $G / H$ is amenable, then $H$ has finite index in $G$. This result is well known in the case where $H$ is a normal subgroup.

\section{Relative Property (T)}

We begin by stating some results on relative property $(T)$ which are direct analogues of existing results for property (T). Since the proofs are minor modifications of those for property $(\mathrm{T})$, we omit the details.

THEOREM 1.1. Let $G$ be a compactly generated locally compact group and let $H$ be a closed subgroup of $G$. The following are equivalent:

(a) The pair $(G, H)$ has property $(T)$.

(b) Every sequence $\left(\phi_{n}\right)_{n \geqslant 1}$ of normalised positive definite functions on $G$ which converges to 1 uniformly on compact subsets of $G$ converges to 1 uniformly on $H$.

(c) Every continuous negative definite function $\psi$ on $G$ is bounded on $H$.

Proof: To prove the equivalence of $(a)$ and $(b)$, imitate [4, Théorème 5.11]. For the proof of the equivalence of $(b)$ and $(c)$ see [1, Theorem 3$]$.

Proposition 1.2. Let $G$ be a locally compact group and let $H$ be a closed subgroup of $G$. If $(G, H)$ has relative property $(T)$ then $H$ is a subgroup of a compactly generated subgroup $G_{0}$ of $G$.

Proof: Compare [4, Théorème 3.4].

REMARK. $H$ need not itself be compactly generated. For example, take $G=S L(3, \mathbb{Z})$. $G$ contains the free group on two generators $\mathbb{F}_{2}$ and $\mathbb{F}_{2}$ contains the free group on infinitely many generators, $H=\mathbb{F}_{\infty}$. Since $G$ has property $(\mathrm{T}),(G, H)$ has relative property (T), but $H$ is not compactly generated.

Proposition 1.3. Let $G$ be a locally compact group and $H$ a normal subgroup of $G$. If $(G, H)$ has property $(T)$ and $G / H$ has (the usual) property (T) then $G$ has property $(T)$.

Proof: Although the statement is rather different, the proof uses the same method as $[4$, Théorème 1.9].

THEOREM 1.4. Let $G$ be a compactly generated locally compact group. Suppose we have closed subgroups $H<K<G$, such that $(G, B)$ has property (T) and 
$K$ has finite covolume in $G$. Then $(K, H)$ has property $(T)$.

Proof: Compare [4, Théorème 3.4].

As a special case, where $K=H$, we have:

Corollary. If $(G, H)$ has property $(T)$ and $H$ has finite covolume in $G$, then $H$ has (the usual) property (T).

TheOREM 1.5. Let $G$ be a compactly generated locally compact group with closed subgroups $H<K<G$. Suppose that $H$ has finite covolume in $K$ and that $(G, H)$ has property $(T)$. Then $(G, K)$ has property $(T)$.

Proof: Compare [8, Theorem III.2.12] or, in the case where $H$ is co-compact in $G,[4$, Théorème 3.12$]$.

As a special case, where $K=G$, we have:

COROLlary. If $H$ is a closed subgroup of finite covolume in $G$ and $(G, H)$ has property $(T)$, then $G$ has (the usual) property $(T)$.

\section{Positive Definite Functions and Subgroups of Discrete Groups}

From now on we shall consider only countable discrete groups, partly because of the nature of our results and partly because the positive definite functions we shall define would otherwise be discontinuous. The following result is used repeatedly.

Proposition 2.1. Let $H$ be a subgroup of a group $G$. If $\phi_{0}$ is a positive definite function on $H$, then the function $\phi$ defined by

$$
\phi(g)= \begin{cases}\phi_{0}(g) & g \in H \\ 0 & g \in G-H\end{cases}
$$

is positive definite on $G$.

Proof: See $[5,32.43]$.

REMARK. In particular, the characteristic function of a subgroup $H, 1_{H}$, is a positive definite function on $G$. This special case has a very simple proof based on the observation that $1_{H}$ is a matrix coefficient of the quasi-regular representation $\lambda_{H}=\operatorname{In} d_{H}^{G} 1_{H}$ of $G$ on the Hilbert space $\ell^{2}(G / H)$. In fact

$$
\lambda_{H}(g) \delta_{x H}=\delta_{g x H}
$$

and

$$
1_{H}(g)=\left\langle\lambda_{H}(g) \delta_{H} \mid \delta_{H}\right\rangle .
$$

These positive definite functions can be used to prove the following theorem. 
Proposition 2.2. If $G$ has property (T) and $n \in \mathbb{N}$ then $G$ has a finite number of subgroups of index $n$.

Proof: $G$ is finitely generated [4, Théorème 1.1] and so the result is a special case of a result of M. Hall [7, Theorem 4.7]. However, we can give a new proof as follows, using the fact that the space $P(G)$ of normalised positive definite functions on $G$ is a compact metric space with the topology of pointwise convergence [1].

If there is an infinite sequence of distinct subgroups $H_{i}$ of index $n$ in $G$, then, by compactness of $P(G)$, the sequence $\phi_{i}=1_{H_{i}}$ has a convergent subsequence $\phi_{i j}$ whose limit is necessarily the characteristic function of a subgroup $H$ of index $\leqslant n$. Now $\boldsymbol{H}$ has property (T) by $[4$, Proposition 3.15] and so convergence is uniform on $H$. This implies that $H_{i j}=H$ for sufficiently large $j$, which is a contradiction.

Proposition 2.1 can be combined with the convergence criterion of Theorem 1.1 to give a proof of Theorems 1.4 and 1.5 in the case of discrete groups. In this case a subgroup of finite covolume is simply one of finite index. The proof of Theorem 1.5 is then relatively straightforward, but Theorem 1.4 requires a new method of extending positive definite functions that we now detail.

Let $H$ be a subgroup of finite index $n$ in $G$ and let $G / H=\left\{g_{1} H, g_{2} H, \ldots, g_{n} H\right\}$ where $g_{1}=1$. Let $\phi$ be a normalised positive definite function on $H$. We show how to extend $\phi$ to a normalised positive definite function $\tilde{\phi}$ on $G$.

Firstly note that if $x \in G$ then, for each $j, x g_{j} \in g_{i} H$ for exactly one $i$ and so

(a) For each $j, g_{i}{ }^{-1} x g_{j} \in H$ for exactly one $i \in\{1,2, \ldots, n\}$ and, taking inverses,

(b) For each $i, g_{i}^{-1} x g_{j} \in H$ for exactly one $j \in\{1,2, \ldots, n\}$.

Now extend $\phi$ to be zero outside $H$. (The resulting function is positive definite by Proposition 2.1.) Define $\tilde{\phi}: G \rightarrow \mathbb{C}$ by

$$
\widetilde{\phi}(x)=\frac{1}{n} \sum_{i, j} \phi\left(g_{i}^{-1} x g_{j}\right)
$$

We claim that

(1) $\tilde{\phi}$ is positive definite and $\tilde{\phi}(1)=1$.

(2) For each $x \in G$ the sum (2.1) contains exactly $n$ nonzero terms.

REMARK. The definition of $\tilde{\phi}$ is analogous to the definition of the induced character in the case of finite groups. Unlike that case however, our $\widetilde{\phi}$ depends on the choice of $g_{j}(2 \leqslant j \leqslant n)$, since $\phi$ is not, in general, a class function.

Claim (2) follows immediately from assertions (a) and (b). 
Proof of Claim (1): $\widetilde{\phi}(1)=1$, since by (a) and (b),

$$
\tilde{\phi}(1)=\frac{1}{n} \sum_{i=1}^{n} \phi\left(g_{i}^{-1} g_{i}\right)=\frac{1}{n} \sum_{i=1}^{n} \phi(1)=1
$$

To prove positive definiteness, let $F$ be a finite subset of $G$ and for each $x \in F$ let $\alpha_{z} \in \mathbb{C}$. We need to show that

$$
\sum_{x, y \in F} \bar{\alpha}_{x} \alpha_{y} \tilde{\phi}\left(x^{-1} y\right) \geqslant 0
$$

For all $i, j \in\{1, \ldots, n\}, g_{i}^{-1} x^{-1} y g_{j} \in H$ if and only if $g_{i}^{-1} x^{-1} g_{k} \in H$ and $g_{k}^{-1} y g_{j} \in H$ for some (unique) $k$. (Note that $g_{i}^{-1} x^{-1} g_{k} \in H$ for a unique $k$ and then $g_{k}^{-1} y g_{j}=\left(g_{i}^{-1} x^{-1} g_{k}\right)^{-1} g_{i}^{-1} x^{-1} y g_{j} \in H$ also. $)$

For the rest of the proof, recall that $\phi=0$ outside $H$. Now

$$
\begin{aligned}
\sum_{x, y \in F} \bar{\alpha}_{x} \alpha_{y} \tilde{\phi}\left(x^{-1} y\right) & =\frac{1}{n} \sum_{x, y \in F} \sum_{i, j} \bar{\alpha}_{x} \alpha_{y} \phi\left(g_{i}^{-1} x^{-1} y g_{j}\right) \\
& =\frac{1}{n} \sum_{k=1}^{n} \sum_{x, y \in F} \sum_{i, j} \bar{\alpha}_{x} \alpha_{y} \phi\left(\left(g_{i}^{-1} x^{-1} g_{k}\right)\left(g_{k}^{-1} y g_{j}\right)\right)
\end{aligned}
$$

and, for each $k$,

$$
\sum_{x, y \in F} \sum_{i, j} \bar{\alpha}_{x} \alpha_{y} \phi\left(\left(g_{k}^{-1} x g_{i}\right)^{-1}\left(g_{k}^{-1} y g_{j}\right)\right) \geqslant 0
$$

since $\phi$ is positive definite on $H$.

We now use the above observations to prove Theorem 1.4 in the discrete case.

THEOREM 2.3. Let $G$ be a finitely generated discrete group with subgroups $H<K<G$. Suppose that $K$ is of finite index in $G$ and that $(G, H)$ has property $(T)$. Then $(K, H)$ has property $(T)$.

Proof: Let $\left(\phi_{i}\right)$ be a sequence of positive definite functions on $K$ such that $\phi_{i} \rightarrow 1$ pointwise on $K$. Define $\tilde{\phi}_{i}$ as above. Then $\tilde{\phi}_{i}$ is positive definite. Also, for each $x \in G, \widetilde{\phi}_{i}(x)$ is by definition the average of $n$ complex numbers in the unit disc, each of which converges to 1 . Therefore $\widetilde{\phi}_{i}$ converges pointwise to 1 on $G$ and, since $(G, H)$ has property $(\mathrm{T}), \tilde{\phi}_{i}$ converges uniformly to 1 on $H$. It follows that each of the non-zero terms converges uniformly to 1 on $H$, and hence $(K, H)$ has property (T).

This method can be used to prove a similar result for the class of groups having the Haagerup approximation property. 
DEFINITION 2.1. A discrete group $G$ has the Haagerup approximation property if $C_{0}(G)$ has an approximate unit consisting of positive definite functions.

Any amenable group has the Haagerup approximation property as does any finitely generated free group [3].

Proposition 2.4. Let $G$ be a discrete group and $H$ a subgroup of finite index $n$ in $G$. Suppose that $H$ has the Haagerup approximation property. Then so does $G$.

Proof: Let $\left(\phi_{i}\right)$ be a sequence of positive definite functions in $C_{0}(H)$ such that $\phi_{i} \rightarrow 1$ pointwise on $H$. Define $\widetilde{\phi}_{i}$ as above. Then $\tilde{\phi}_{i}$ is positive definite and vanishes at $\infty$. Also for each $x \in G, \widetilde{\phi}_{i}(x)$ is by definition the average of $n$ complex numbers in the unit disc, each of which converges to 1 . Therefore $\tilde{\phi}_{i}$ converges pointwise to 1 . [

The interest of the Haagerup approximation property for relative property $(T)$ is provided by the following.

PROPOSITION 2.5. Let $G$ be a discrete group with the Haagerup approximation property and let $H$ be a subgroup of $G$ such that $(G, H)$ has property $(T)$. Then $H$ is finite.

Proof: Since $G$ has the Haagerup approximation property, it follows from condition (3B) of [1] that there exists a negative definite function $\psi$ on $G$ such that $\psi(g) \rightarrow \infty$ as $g \rightarrow \infty$. (That is, for each $M \geqslant 0$ there exists a finite subset $F$ of $G$ such that $\psi(g)>M$ for all $g \in G-F$.)

If $H$ is an infinite subgroup of $G$ then $\left.\psi\right|_{H}$ is unbounded and so by Theorem 1.1(c), $(G, H)$ does not have property $(\mathrm{T})$.

\section{REMARKS.}

(1) In particular, $G$ has no infinite subgroups $B$ with the (usual) property (T).

(2) We do not know whether the result of Proposition 2.5 characterises groups $G$ with the Haagerup approximation property.

\section{AMENABLE QUOTIENTS}

If a discrete group $G$ has Kazhdan's property $(\mathrm{T})$ and $H$ is a normal subgroup of $G$ then $G / H$ also has property (T) [4, Proposition 1.6]. It follows that if the group $G / H$ is amenable then it must be finite [4, Proposition 1.7 (i)].

We now extend this result to the case when the subgroup $B$ is not normal. Our result is folklore in the area. It is, for example, essentially stated on page 18 of [2]. (We are grateful to $A$. Valette for pointing out this reference.) However we believe that it is worth giving a complete exposition. 
The first problem is to decide on what it means for $G / H$ to be amenable, since it is no longer a group.

Let $G$ be a finitely generated group with finite symmetric generating set $S$. The Schreier coset graph (which we denote by $G / H$ ) has as its vertices elements of $G / H$. The edges of the graph join $g H$ to $s g H$ whenever $s \in S$. See [7, page 163], but note that we have used left cosets in our definition.

Now let $X$ be any graph with vertex set $X^{0}$ and edge set $X^{1}$. If $F \subset X$, the boundary $\partial F$ of $F$ is the set of edges $b \in X^{1}$ having one end in $F$ and the other end in $X-F$. The isoperimetric number of the graph $X$ is

$$
i(X)=\inf \left\{\frac{|\partial F|}{|F|}: F \subset X, F \text { finite }\right\} .
$$

If $X$ has bounded degree (as is the case for a Schreier coset graph) then we say that $X$ is amenable if $i(X)=0$ [9, Section 5]. If $X$ is the Cayley graph of a finitely generated group $G$ then this coincides with the usual definition of amenability for $G$. In that case the condition $i(G)=0$ is simply a restatement of the Følner condition [10, Chapter 4].

We require one more graph-theoretic concept. Choose an arbitrary orientation for the graph $X$, so that each $b \in X^{1}$ has initial vertex $o(b)$ and final vertex $e(b)$. The simplicial coboundary operator $d: \ell^{2}\left(X^{0}\right) \rightarrow \ell^{2}\left(X^{1}\right)$ is defined by $d f(b)=f(e(b))-f(o(b))$. The combinatorial Laplacian of the graph $X$ is $\Delta=d^{*} d . \Delta$ does not depend on the chosen orientation because of the formula

$$
\Delta f(x)=v(x) f(x)-\sum f(y)
$$

where $f \in \ell^{2}\left(X^{0}\right), v(x)$ is the number of vertices $y$ adjacent to $x$ and the sum is over all such $y[4$, page 98$]$.

Now suppose $X$ is the Schreier coset graph $G / H$. Then $\Delta=\sum_{s \in S}\left(1-\lambda_{H}(s)\right)$, where $\lambda_{H}$ is the quasi-regular representation of $G$ on $\ell^{2}(G / H)$.

THEOREM 3.1. Suppose that $G$ is a discrete group with property $(T)$ and let $S$ be a finite symmetric set of generators for $G$. Let $H$ be a subgroup such that the graph $G / H$ is amenable. Then $H$ has finite index in $G$.

Proof: The basic idea is similar to the proof of [11, Theorem B]. Let $\varepsilon>0$ as in the definition of property $(T)$. (See the introduction.)

Since $G / H$ is amenable, there exists a finite subset $F$ of $G / H$ such that $|\partial F| /|F|<\varepsilon^{2} / 2$ Let $\xi=1_{F} \in \ell^{2}(G / H)$. Then

$$
\frac{\|d \xi\|^{2}}{\|\xi\|^{2}}=\frac{|\partial F|}{|F|}<\frac{\varepsilon^{2}}{2} .
$$


Let $\eta=\|\xi\|^{-1} \xi$. Then $\langle\Delta \eta \mid \eta\rangle=\left\langle d^{*} d \eta \mid \eta\right\rangle=\|d \xi\|^{2} /\|\xi\|^{2}<\varepsilon^{2} / 2$. Equivalently, $\sum_{s \in S}\left\langle 1-\lambda_{H}(s) \eta \mid \eta\right\rangle<\varepsilon^{2} / 2$, so that $\operatorname{Re}\left\langle\eta-\lambda_{H}(s) \eta \mid \eta\right\rangle<\varepsilon^{2} / 2$ for all $s \in S$. That is $\left\|\eta-\lambda_{H}(s) \eta\right\|^{2}=2 R e\left\langle\eta-\lambda_{H}(s) \eta \mid \eta\right\rangle\left\langle\varepsilon^{2}\right.$ for all $s \in S$.

This shows that $\lambda_{H}$ almost has invariant vectors and so, by property $(\mathrm{T}), \lambda_{H}$ has a unit invariant vector $\eta_{0} \in \ell^{2}(G / H)$. Since $G$ acts transitively on $G / H, \eta_{0}$ must be a constant function. Then $\eta_{0} \in \ell^{2}(G / H)$ implies that $|G / H|<\infty$ as required.

RemarK. Amenability for a Schreier coset graph $G / H$ is independent of the finite symmetric generating set $S$ for $G$.

For if $S_{1}, S_{2}$ are two such sets of generators, there exists a number $N$ such that every element of $S_{1}$ is the product of at most $N$ elements of $S_{2}$ and every element of $S_{2}$ is the product of at most $N$ elements of $S_{1}$. It follows that

$$
\frac{1}{N} d_{1}(x, y) \leqslant d_{2}(x, y) \leqslant N d_{1}(x, y)
$$

where $d_{1}, d_{2}$ are the distances between vertices of $G / H$ relative to $S_{1}, S_{2}$ respectively. The identity map is therefore a rough isometry, in the sense of [6], with respect to these two distances. Now rough isometries preserve the strong isoperimetric inequality [6, Proposition 2.1], so the condition $i(G)=0$ is independent of the choice of generators. We are grateful to M. Soardi for kindly showing us this fact.

\section{REFERENCES}

[1] C.A. Akemann and M.E. Walter, 'Unbounded negative definite functions', Canad. J. Math. 33 (1981), 862-871.

[2] M. Gromov, 'Asymptotic invariants of infinite groups', in Geometric Group Theory, (G.A. Niblo and M.A. Roller, Editors), L.M.S. Lecture Note Series 182 (Cambridge University Press, 1993).

[3] U. Haagerup, 'An example of a non nuclear $\mathrm{C}^{*}$-algebra which has the metric approximation property', Invent. Math. 50 (1979), 279-293.

[4] P. de la Harpe and A. Valette, 'La propriété (T) de Kazhdan pour les groupes localement compacts', Astérisque 175 (1989).

[5] E. Hewitt and K. Ross, Abstract harmonic analysis II (Springer-Verlag, Berlin, Heidelberg, New York, 1970).

[6] M. Kanai, 'Analytic inequalities, and rough isometries between noncompact Riemannian manifolds', in Curvature and topology of Riemannian manifolds, (T. Taniguchi, K. Shiohama, T. Sakai and T. Sunada, Editors), Lecture Notes in Math. 1201 (Springer-Verlag, Berlin, Heidelberg, New York, 1986), pp. 123-137.

[7] R.C. Lyndon and P.E. Schupp, Combinatorial group theory (Springer-Verlag, Berlin, Heidelberg, New York, 1977). 
[8] G.A. Margulis, Discrete subgroups of semisimple Lie groups (Springer-Verlag, Berlin, Heidelberg, New York, 1991).

[9] B. Mohar and W. Woess, 'A survey on spectra of infinite graphs', Bull. London Math. Soc. 21 (1989), 209-234.

[10] A.L.T. Paterson, Amenability, Math. Surveys and Monographs 29 (Amer. Math. Soc., 1988).

[11] G. Stuck, 'Growth of homogeneous spaces, density of discrete subgroups and Kazhdan's property (T)', Invent. Math. 109 (1992), 505-517.

Department of Mathematics

University of Ottawa

Ontario K1N 6N5

Canada

e-mail: 060502@acadvml.uottawa.ca
Department of Mathematics

University of Newcastle

New South Wales 2308

Australia

e-mail: guyan@frey.newcastle.edu.au 